\title{
Molecular mechanism of Epicedium treatment for depression based on network pharmacology and molecular docking technology
}

Yankai Dong ${ }^{1 \dagger}$, Bo Tao $^{2 \dagger}$, Xing Xue ${ }^{1}$, Caixia Feng ${ }^{1}$, Yating Ren ${ }^{1}$, Hengyu Ma', Junli Zhang ${ }^{1}$, Yufang Si ${ }^{1}$, Sisi Zhang ${ }^{1}$, Si Liu', Hui Li ${ }^{3}$, Jiahao Zhou', Ge Li', Zhifei Wang ${ }^{1}$, Juanping Xie ${ }^{4^{*}}$ and Zhongliang Zhu ${ }^{1 *}$

\begin{abstract}
Background: Increasing attention has been paid to the effect of Epimedium on the nervous system, particularly anti-depression function. In the present study, we applied network pharmacology to introduce a testable hypothesis on the multi-target mechanisms of Epicedium against depression.

Methods: By reconstructing the network of protein-protein interaction and drug-component-target, we predicted the key protein targets of Epicedium for the treatment of depression. Then, through molecular docking, the interaction of the main active components of Epicedium and predicted candidate targets were verified.

Results: Nineteen active compounds were selected from Epicedium. There were 200 targets associated with Epicedium and 537 targets related to depression. The key targets of Epicedium for treating depression were IL6, VEGFA, AKT1, and EGF. According to gene ontology functional enrichment analysis, 22 items of biological process (BP), 13 items of cell composition (CC) and 9 items of molecular function (MF) were obtained. A total of 56 signaling pathways $(P<0.05)$ were identified by Kyoto Encyclopedia of Genes and Genomes analysis, mainly involving depression-related pathways such as dopaminergic synapse, TNF signaling pathway, and prolactin signaling pathway. The results of molecular docking showed that the most important activity components, including luteoklin, quercetin and kaempferol, were well combined with the key targets.
\end{abstract}

Conclusions: Luteoklin, quercetin, kaempferol and other active compounds in Epicedium can regulate multiple signaling pathways and targets such as IL6, AKT1, and EGF, therefore playing therapeutic roles in depression.

Keywords: Epimedium, depression, network pharmacology, molecular docking, pathway analysis

\footnotetext{
*Correspondence: xjp_731205@163.com; zlzhu@nwu.edu.cn

${ }^{+}$Yankai Dong and Bo Tao contributed equally to this work.

${ }^{4}$ Qinba Chinese Medicine Resources R\&D Center, School of Medicine, Ankang

University, Ankang 710069, Shanxi Province, China

'Key Laboratory of Resource Biology and Biotechnology in Western China,

Ministry of Education, College of Life Sciences, Institute of Maternal and Infant health, Northwest University, Xi'an 710069, Shanxi Province, China

Full list of author information is available at the end of the article
}

(c) The Author(s). 2021 Open Access This article is licensed under a Creative Commons Attribution 4.0 International License, which permits use, sharing, adaptation, distribution and reproduction in any medium or format, as long as you give appropriate credit to the original author(s) and the source, provide a link to the Creative Commons licence, and indicate if changes were made. The images or other third party material in this article are included in the article's Creative Commons licence, unless indicated otherwise in a credit line to the material. If material is not included in the article's Creative Commons licence and your intended use is not permitted by statutory regulation or exceeds the permitted use, you will need to obtain permission directly from the copyright holder. To view a copy of this licence, visit http://creativecommons.org/licenses/by/4.0/ The Creative Commons Public Domain Dedication waiver (http://creativecommons.org/publicdomain/zero/1.0/) applies to the data made available in this article, unless otherwise stated in a credit line to the data. 


\section{Background}

Depression is a common chronic and highly disabling disorder with high level of treatment resistance [1]. Depression has affected more than 350 million people worldwide [2-4], and it is characterized by weight loss, low energy, loss of appetite, and insomnia [5, 6]. In recent years, most antidepressants have become NMDA receptor antagonists, which can produce antidepressant effects quickly, but these drugs are not used as first-line antidepressants because of their side effects, such as sensory agitation, cognitive impairment, addiction and hallucination [7, 8]. In addition, the pathogenesis of depression has been partly elucidated based molecular and genetic studies, but the potential mechanism of depression needs to be determined [9].

The primary task of prevention and treatment of depression is the development of new antidepressant therapeutic targets and therapeutic drugs with short onset latency and less side effects. Considering the improved safety and fewer side effects, traditional Chinese medicines (TCMs) performs an important role in the prevention and treatment of diseases [10], and have increasing attention among scientists worldwide. TCMs have multiple ingredients, targets and ways to be effective [11]. As a Chinese herbal medicine, Epicedium is well-known for wide-ranging effects, such as treatment of cancer, cardiovascular diseases, and immune suppression [12]. The extract of Epicedium and its component icariin can effectively promote the regeneration of peripheral nerve and improve the damaged nerve function [13]. Epicedium ethanol extraction exerts an anti-inflammatory effect by inhibiting the production of tumor necrosis factor- $\alpha$, interleukin (IL)-1 $\beta$, and IL-6 in lipopolysaccharide(LPS)-induced peritonitis [14]. Icariin can ameliorate depressive behavior of male offspring with prenatal stress [15]. In addition, Epicedium is rich in chemical composition, including flavonoids, lignans, and polysaccharides [12]. However, the therapeutic mechanism of Epicedium on depression is not clear.

With the rapid progress of bioinformatics, systems biology, and poly-pharmacology, network-based drug discovery has become a promising approach for the development of effective drugs. In 2007, Hopkins et al. first proposed the concept "network pharmacology". This method analyzes the intervention of drugs and potential treated targets of diseases based on system biology [16, 17]. Network pharmacology highlights a paradigm shift from the current "one target, one drug" strategy to a novel version of the "network target, multi-component" strategy [18]. In TCM research, it is widely used because of the integrity and system consistent with the TCM prescription's principles $[19,20]$. As a computer-aided drug design method that depending on the interaction and affinity between the target and active compound, molecular docking has been widely used in the material basis of TCMs [21].

In the present study, we applied network pharmacology and molecular docking to clarify the mechanism of Epicedium in the treatment of depression. We systematically analyzed the active ingredients, potential targets, pathways and networks affected by Epicedium for the treatment of depression. We also performed molecular docking studies to predict the interactions that allow important compounds to bind to its predicted targets. Our results may help clarify how Epicedium can be effective against depression and facilitate the development of novel drugs.

\section{Methods \\ Prediction of target genes associated with depression}

Genes related to depression were obtained from the National Center for Biotechnology Information database (https://www.ncbi.nlm.nih.gov/) through searching "depression". After filtering with the term "Homo sapiens", 537 genes associated with depression were identified in the Gene Bank database.

\section{Collection of component targets of Epicedium}

The compounds and targets of Epicedium were mainly collected from TCMSP, a natural product database for Chinese herbal medicines (http://ibts.hkbu.edu.hk/LSP/ tcmsp.php,updated on May 31, 2014 )[22]. In clinical treatment, TCMs are often used via oral administration. Oral bioavailability (OB)] [23] and drug-likeness (DL )[24], which are ADME-related models, mainly affect the absorption of drugs by the gastrointestinal tract. Therefore, we screened bioactive components under the standards of $\mathrm{OB} \geq 30 \%$ and $\mathrm{DL} \geq 0.18$ [25], and obtained the related targets of each components. The common targets of Epicedium and the depression related genes were intersected with the Venn map. The intersected genes were the target genes of Epicedium for the treatment of depression.

\section{Protein-protein Interaction (PPI) Data}

String database (https://string-db.org/cgi/input.pl) [26] contains known and predicted protein-protein interaction. A large number of PPIs were collected involving 9643763 proteins and 1380838440 interactions, including data obtained from experimental detection and bioinformatics prediction. The intersected genes were imported into the string database. The species was defined as "Homo sapiens". The PPI data was obtained. The results were saved in TSV format. The information of node1, node 2 and combined score in the file were retained. The interaction network was drawn and the network was analyzed by cytoscape. 


\section{Network Construction}

The active components of Epicedium and intersected genes were imported into Cytoscape version 3.6.0 to construct the compound-target network of Epicedium.

\section{Gene Ontology (GO) and Pathway Analysis}

We used the Database for Annotation, Visualization, and Integrated Discovery [27] (DAVID, https://david. ncifcrf. gov/, v6.8) database for GO enrichment analysis and accomplished pathway enrichment analysis by using Kyoto Encyclopedia of Genes and Genomes [28](KEGG, http://www.kegg.jp/). Biological information annotation database (David, https://david. ncifcrf. gov/, version 6.8) provides a systematic and comprehensive annotation information on the biological functions of large-scale genes or proteins, and it can determine the most considerably enriched biological annotation. The insected genes were imported into the David database. The selected identifier was set to official gene symbol. The list type was set to gene list, and the species was limited to "Homo sapiens". Go analysis and KEGG pathway analysis were performed on the insected genes.

\section{Molecular docking}

The crystal structures of the candidate protein targets of Epicedium were downloaded from the RCSB Protein Data Bank (http://www.pdb.org/) and modified using the Autodock tools 1.5.6 software. The four targets were IL6 (PDB ID: 5fuc )[29], VEGFA (PDB ID: 6d3o; https:// www1.rcsb.org/structure/6d3o), AKT1(PDB ID: 6s9x) [30], and EGF (PDB ID:1j19) )[31]; these targets include ligand and water removal, hydrogen addition, and amino acid optimization and patching. The files were saved in pdbqt format. ChemBioDraw 3D was used to create the 3D chemical structures and minimize their energy. Results were saved in MOL.2 format. The compounds were imported into Autodock tools 1.5.6, and all flexible keys were rotatable by default and saved in pdbqt format, as docking ligand. Autodock Vina 1.1.2 was used for docking, while Discovery Studio 3.5 was used to visualize the docking results.

\section{Results}

\section{Active compounds of Epicedium}

TCMSP database (http://ibts.hkbu.edu.hk/LSPtcmsp. php) is a unique pharmacological platform of TCM system, and it can calculate absorption, distribution, metabolism and excretion (ADME)-related characteristics of natural compounds [22]. A total of $130 \mathrm{com}-$ ponents of Epicedium were identified from TCMSP. The components were screened with the criteria of $\mathrm{OB} \geq 30 \%$ and $\mathrm{DL} \geq 0.18$. A total of 23 bioactive components of Epicedium were screened, in which four had no targets. Finally, 19 compounds were collected from TCMSP database (Fig. 1A, B; Table 1).

\section{Depression-Epicedium PPI network}

By using depression as keyword in NCBI database, 537 genes related to depression were retrieved, and 200 target genes of Epicedium were collected from the TCMSP database. After intersecting the target genes of Epicedium and depression, 53 common genes were found (Fig. 2A). These genes could be the target genes of Epicedium in the treatment of depression. Then, we built a visualized Epicedium-component-targets network by using Cytoscape 3.6. The nodes of different colors and shapes represented the

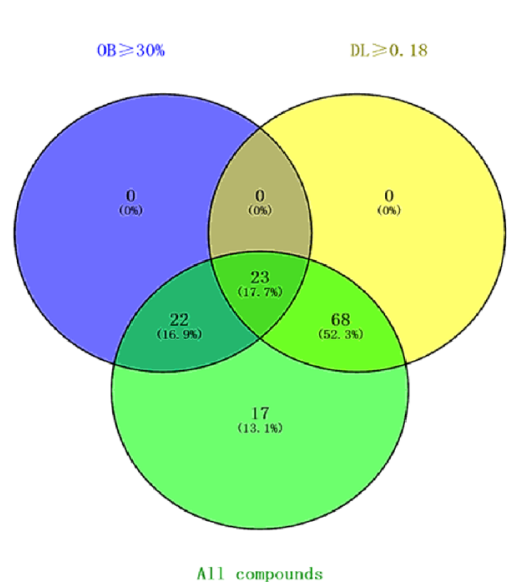

A

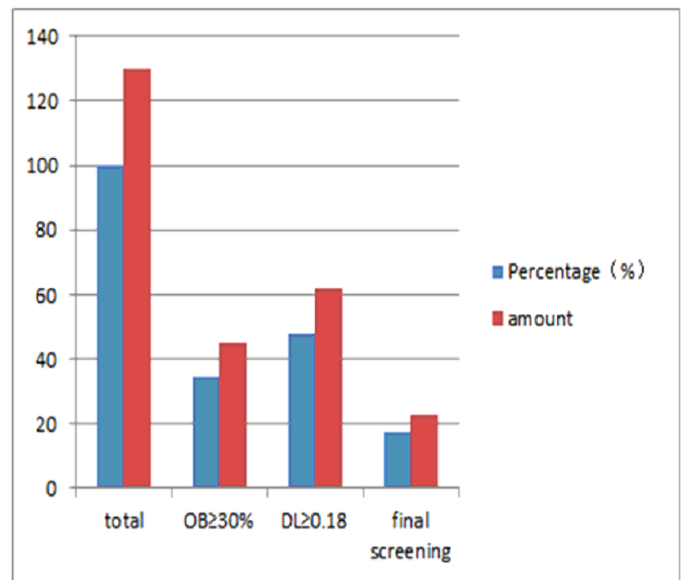

B

Fig. 1 Epimedium screening of bioactive compounds. (A) Venn diagram: 130 components (green section) and 23 bioactive components screened by two ADME-related models (blue section stands for the components of $O B \geq 30 \%$, yellow section stands for $D L \geq 0.18$ ). (B) Distributions of components in Epimedium 
Table 1 Basic information about the active compounds in Epimedium (OB $\geq 30 \%, D L \geq 0.18$ )

\begin{tabular}{|c|c|c|c|c|}
\hline N.O. & Molld & MolName & $\mathrm{OB}(\%)$ & $\mathrm{DL}$ \\
\hline 1 & MOL001645 & Linoleyl acetate & 42.10 & 0.20 \\
\hline 2 & MOL001792 & DFV & 32.76 & 0.18 \\
\hline 3 & MOL003044 & Chryseriol & 35.85 & 0.27 \\
\hline 4 & MOL003542 & 8-Isopentenyl-kaempferol & 38.04 & 0.39 \\
\hline 5 & MOL000359 & sitosterol & 36.91 & 0.75 \\
\hline 6 & MOL000422 & kaempferol & 41.88 & 0.24 \\
\hline 7 & MOL004367 & olivil & 62.23 & 0.41 \\
\hline 8 & MOL004373 & Anhydroicaritin & 45.41 & 0.44 \\
\hline 9 & MOL004380 & C-Homoerythrinan, 1,6-didehydro-3,15,16-trimethoxy-, (3.beta.)- & 39.14 & 0.49 \\
\hline 10 & MOL004382 & Yinyanghuo A & 56.96 & 0.77 \\
\hline 11 & MOL004384 & Yinyanghuo C & 45.67 & 0.50 \\
\hline 12 & MOL004388 & 6-hydroxy-11,12-dimethoxy-2,2-dimethyl-1,8-dioxo-2,3,4,8-tetrahydro-1H-isochromeno[3,4-hisoquinolin-2-ium & 60.64 & 0.66 \\
\hline 13 & MOL004391 & 8-(3-methylbut-2-enyl)-2-phenyl-chromone & 48.54 & 0.25 \\
\hline 14 & MOL004396 & 1,2-bis(4-hydroxy-3-methoxyphenyl)propan-1,3-diol & 52.31 & 0.22 \\
\hline 15 & MOL004427 & Icariside A7 & 31.91 & 0.86 \\
\hline 16 & MOL000006 & luteolin & 36.16 & 0.25 \\
\hline 17 & MOL000622 & Magnograndiolide & 63.71 & 0.19 \\
\hline 18 & MOL000098 & quercetin & 46.43 & 0.28 \\
\hline 19 & MOL004386 & Yinyanghuo E & 51.63 & 0.55 \\
\hline
\end{tabular}

potential active components and targets of Epicedium. The blue nodes represented Epicedium, the yellow nodes represented the active components of Epicedium, and the red nodes represented the potential antidepressant targets of Epicedium. The edges represented the correlation between the active components and the targets (Fig. 2B), confirming the multicomponent and multi-target characteristics of Epicedium. A total of 199 nodes and 3,302 edges were observed in Fig. $2 \mathrm{C}$, the average node degree was 33.2, and the local clustering coefficient was 0.574 . A total of 53 nodes and 449 edges were observed in Fig. 2D, the average node degree was 16.9 , and the local clustering coefficient was 0.662 . The size of the node represented the degree value of targets. The larger the node was, the greater the degree value was. The thickness of the edge indicated the combination score. The coarser the edge was, the greater the combination score value was. Data for the degree of each target was shown in Table 2.

\section{GO analysis of genes targeted by Epicedium}

David database was applied to analyze the related targets of Epicedium for the treatment of depression. In BP analysis, the top ranked targets were distributed in the process of cell division (5 targets/5.9\%), positive regulation of sequence-specific DNA binding transcription factor activity (7 targets/8.3\%) and transcription from RNA polymerase II promoter (13 targets/15.3\%), response to hypoxia (6 targets/7.1\%), and immune response (7 targets/8.3\%) (Fig. 3A). In CC analysis (Fig. 3B), cell components such as extracellular space (18 targets/21.3\%), cytosol (11 targets/13.0\%), extracellular matrix (4 targets/4.7\%), neuron projection (4 targets/4.7\%), integral component of plasma membrane(8 targets/9.4\%), and extracellular space(18 targets/21.3\%) were at the top. In MF analysis (Fig 3C), molecular functions such as cytokine activity (6 targets/12.8\%), dopamine (3 targets/ $6.4 \%)$ IL-1 receptor (3 targets/6.4\%), steroid (3 targets/ 6.4\%), and drug binding (3 targets/6.4\%), and cytokine activity ( 6 targets/12.8\%) were at the top.

\section{KEGG analysis of genes encoding proteins targeted by Epicedium}

David database was used to analyze the KEGG pathway of 53 targets of Epicedium for the treatment of depression. According to the $\mathrm{p}$ value, the top 20 pathways were selected and shown in Fig. 3D. The color of the nodes in the figure from green to red reflected the $\mathrm{p}$ value from large to small. The nodes from small to large reflected the number of related genes in increasing trend. The top ranked pathways were dopaminergic synapse, measles pathways in cancer, inflammatory bowel disease, leishmaniasis, and chagas disease (American trypanosomiasis). 


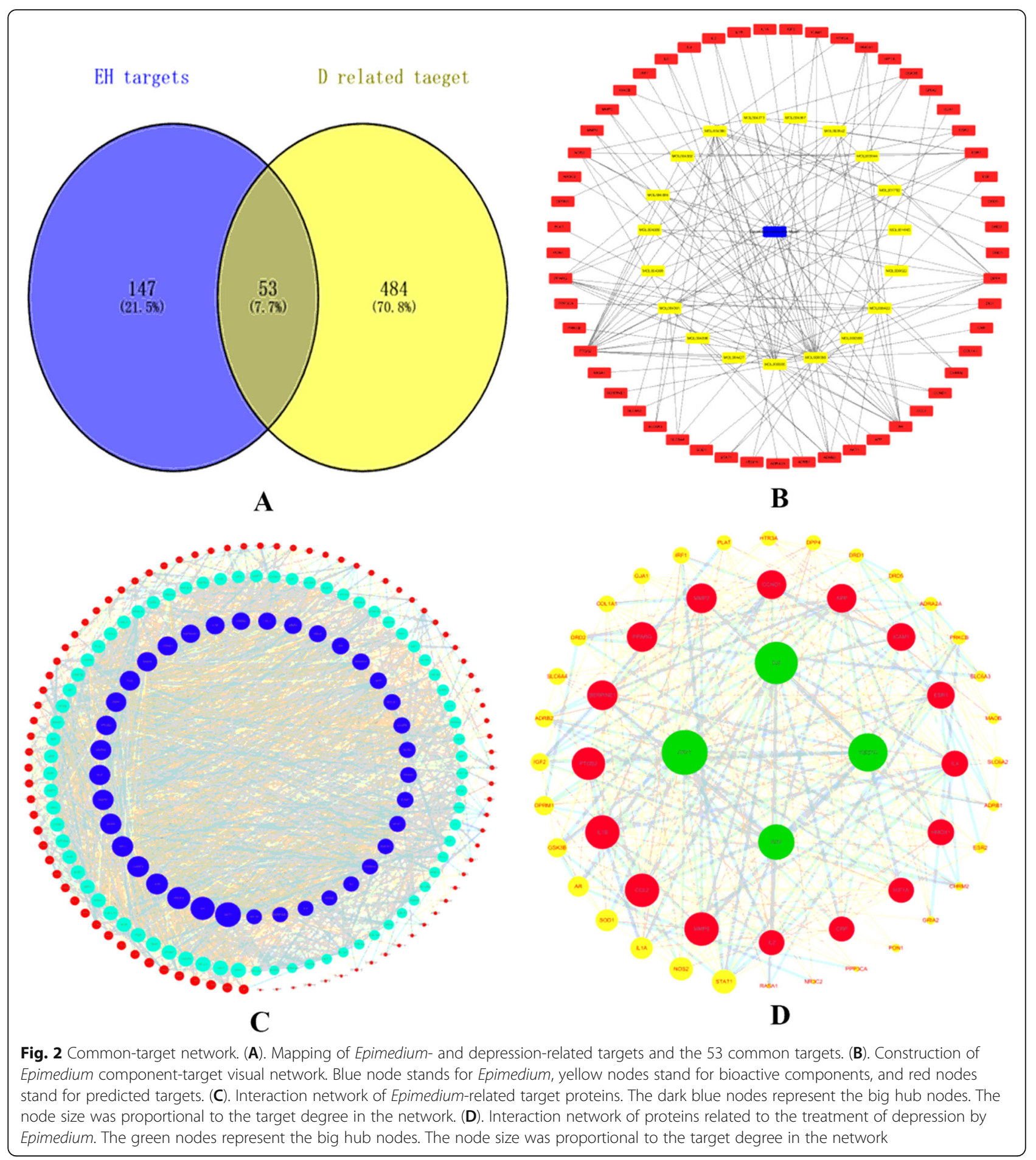

\section{Molecular docking}

The results are shown in Fig. 5 and supplementary Figure1-2. The 2D and 3D structures of the ligands are shown in Fig. 4. The estimated free energy of binding and RMSD was summarized in Table 3. The interactions between ligands and target proteins is shown in Table 4 .
Electrostatic force and van der Waals force are the main forces between ligand and target protein. The binding energies of ligand and receptor were less than -1.19cal / mol, and RMSD were less than 2, indicating that the docking results were good. Fluoxetine was used as positive control, and the binding energies of kaempferol, 
Table 2 Information about potential antidepressant targets from Epimedium

\begin{tabular}{|c|c|c|c|c|c|}
\hline serial number & name & degree & serial number & name & degree \\
\hline 1 & AKT1 & 41 & 27 & OPRM1 & 15 \\
\hline 2 & IL6 & 38 & 28 & IGF2 & 14 \\
\hline 3 & VEGFA & 34 & 29 & ADRB2 & 14 \\
\hline 4 & EGF & 31 & 30 & DRD2 & 13 \\
\hline 5 & IL1B & 29 & 31 & IRF1 & 13 \\
\hline 6 & CCL2 & 29 & 32 & COL1A1 & 13 \\
\hline 7 & MMP9 & 29 & 33 & SLC6A4 & 13 \\
\hline 8 & PTGS2 & 28 & 34 & GJA1 & 13 \\
\hline 9 & SERPINE1 & 25 & 35 & PLAT & 12 \\
\hline 10 & MMP2 & 25 & 36 & HTR3A & 12 \\
\hline 11 & PPARG & 25 & 37 & DPP4 & 12 \\
\hline 12 & CCND1 & 24 & 38 & PRKCB & 11 \\
\hline 13 & APP & 24 & 39 & ADRA2A & 11 \\
\hline 14 & ICAM1 & 23 & 40 & DRD5 & 11 \\
\hline 15 & ESR1 & 22 & 41 & DRD1 & 11 \\
\hline 16 & IL4 & 21 & 42 & SLC6A3 & 9 \\
\hline 17 & HMOX1 & 21 & 43 & ESR2 & 9 \\
\hline 18 & HIF1A & 20 & 44 & ADRB1 & 9 \\
\hline 19 & IL2 & 20 & 45 & SLC6A2 & 9 \\
\hline 20 & CRP & 20 & 46 & MAOB & 9 \\
\hline 21 & STAT1 & 19 & 47 & GRIA2 & 7 \\
\hline 22 & NOS2 & 18 & 48 & CHRM2 & 7 \\
\hline 23 & IL1A & 17 & 49 & PON1 & 7 \\
\hline 24 & SOD1 & 17 & 50 & PPP3CA & 5 \\
\hline 25 & $A R$ & 16 & 51 & RASA1 & 4 \\
\hline 26 & GSK3B & 15 & 52 & NR3C2 & 4 \\
\hline
\end{tabular}

quercetin and luteolin were lower than that of fluoxetine, indicating that the binding stability of kaempferol, quercetin and luteolin was better than that of fluoxetine.

\section{Discussion}

Depression is a common mental disorder with a high incidence, recurrence rate, and mortality. Depression not only seriously endangers the lives and health of the people but also causes heavy mental and economic burden to the society, patients, and families. The pathogenesis of depression is very complex. Depression has become a prevalent worldwide health concern. It is still mainly treated with drugs.

Epicedium has different effects, such as antiinflammatory, anti-aging, anti-tumor, anti-oxidation, and anti-inflammatory effects, improves immunization, and acts as antidepressants. In the present study, the main research idea was based on the perspective of diseasetarget-drug, and we applied the network pharmacology approach to evaluate the anti-depression effects and underlying mechanism of Epicedium. These results provide an important reference for the further study of the pathogenesis of depression and the treatment of depression.

By constructing the "drug-target-disease" network, novel drugs and treatment targets could be found [32]. TCMs are difficult to study because of their complex components and huge system. Network pharmacology provides a new idea and perspective for the study of complex TCMs. We integrated information from open databases to predict the interaction between Epicedium and its potential protein targets in depression. In the present study, Epicedium works in various ways through the effects of multiple compounds and may provide advantages by reducing drug resistance. The results of PPI network show a relationship between the target proteins of Epicedium, which was a complex interaction network rather than acting alone. Nineteen active components were obtained by screening, and the three active components with more targets were luteolin (15 targets), quercetin (34 targets), and kaempferol (14 targets). is the results are similar to Naijun Yuan's results, confirming indicates that flavonoids have obvious antidepressant effect. These flavonoids are common components in many herbal medicines. Their specific roles in the treatment of depression should be clarified [33]. Many studies have demonstrated their neuroprotective effects in vitro and in vivo. For example, luteolin-regulated genes may act as receptors in the central nervous system to produce antidepressant effects [34, 35]. Quercetin has antioxidant and anti-inflammatory effects in the treatment of neurological diseases. Quercetin alleviates anxiety and depression in chronically stressed rats by protecting neurons from oxidation and inflammation [36]. A study on neuroinflammation induced by lipopolysaccharide in mice indicated that quercetin still can eliminate inflammation by significantly reducing the proliferation of astrocytes in the brain and reducing the expression of inflammatory factors [37]. In addition, quercetin can inhibit neuronal apoptosis by regulating the expression of AKT1 and ASK1/JNK3/Caspase-3, thus preventing anxiety behavior. In the future research, new antidepressant drugs can be developed based on the three compounds.

Previous studies have shown that kaempferol [38], luteolin [39] and quercetin [40] have good antidepressant effects. Quercetin and luteolin have short administration time and low dose, which may be the most impotent for antidepressant property.

The molecular weight of quercetin is 302.24 and its melting point is $314-317{ }^{\circ} \mathrm{C}$, water solubility $<0.1 \mathrm{~g} / 100$ $\mathrm{ml}$ at $21{ }^{\circ} \mathrm{C}$. The number of hydrogen bond donors is 5 , the number of hydrogen bond acceptors is 7, the number of rotatable chemical bonds is 1 , the number of 


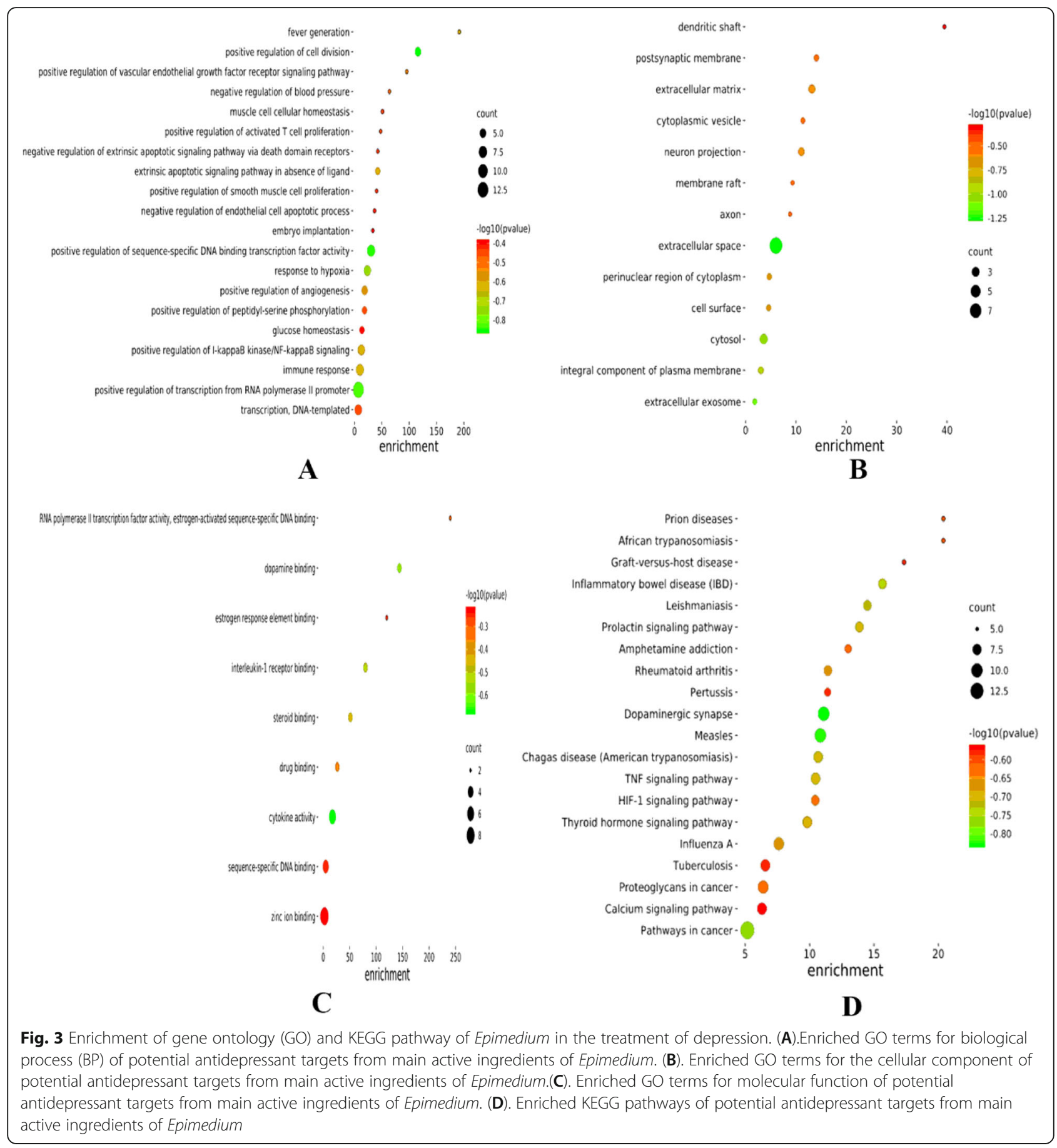

tautomers is 435 , the topological molecular polar surface area is 127 , the number of heavy atoms is 22 , the complexity is 488 , and the number of covalent bond units is 1 . The kinetic process of quercetin in rats is a two compartment open model with absorption, $\mathrm{t} 1 / 2(\alpha)=0.19 \mathrm{~h}$, $\mathrm{t} 1 / 2(\beta)=1.22 \mathrm{~h}, \mathrm{tmax}=0.333 \mathrm{~h}[41]$. Quercetin was quickly distributed to tissues after it was orally given to rats. The highest concentration of quercetin was observed in stomach in rats, and in order of plasma,liver,kidney,heart,lung,spleen. However, the quercetin concentration in muscle and brain couldn't be detected by HPLC [42].

The molecular weight of kaempferol is 286.236 and the melting point is $276{ }^{\circ} \mathrm{C}$. The solubility in ethanol is $20 \mathrm{mg} / \mathrm{ml}$. The number of hydrogen bond donors is 4 , the number of hydrogen bond acceptors is 6 , the number of rotatable chemical bonds is 1 , the number of tautomers is 126 , the polar surface area of topological 


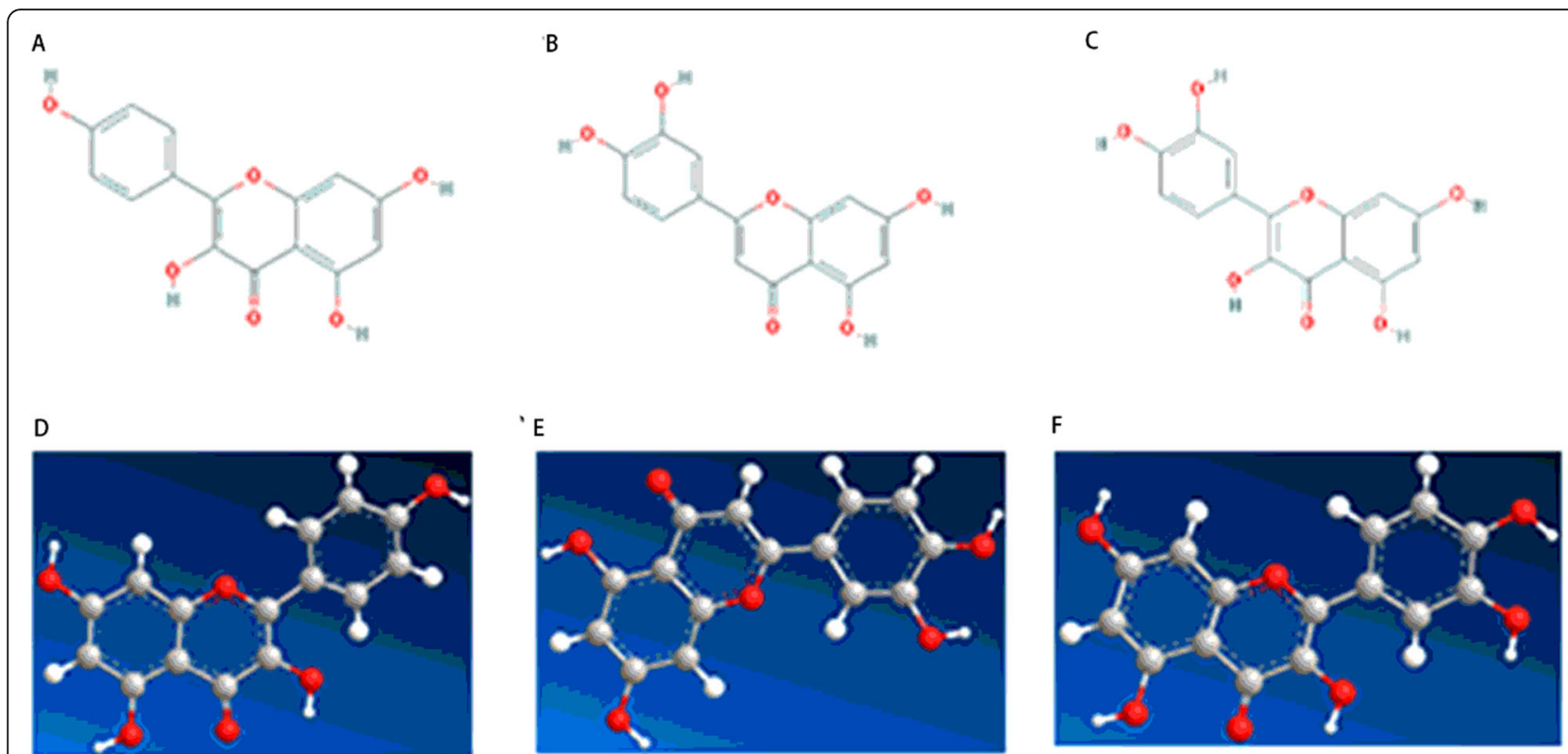

Fig. 4 2D and 3D structures of ligands. (A) 2D structure of kaempferol. (B) 2D structure of luteolin. (C) 2D structure of quercetin. (D) 3D structure of kaempferol. (E) 3D structure of luteolin. (F) 3D structure of quercetin

molecules is 107 , the number of heavy atoms is 21 , the complexity is 451 , the number of covalent bond units is 1. The pharmacokinetic behavior of kaempferol conformed to the two compartment model, $t 1 / 2(\alpha)=0.957 \pm$ $0.172 \mathrm{~min}, \mathrm{t} 1 / 2(\beta)=6.409 \pm 1.584$. Kaempferol has the characteristics of slow absorption, wide distribution and rapid elimination [43].

The molecular weight of luteolin is 286.236 and the melting point is $330{ }^{\circ} \mathrm{C}$. The number of hydrogen bond donors is 4 , the number of hydrogen bond acceptors is 6 , the number of rotatable chemical bonds is 1 , the number of tautomers is 162 , the topological molecular polar surface area is 107 , the number of heavy atoms is 21 , the surface charge is 0 , the complexity is 447 , the number of isotopic atoms is 0 , the number of covalent bond units is 1 , and the solubility is not determined, $\mathrm{t} 1 / 2(\alpha)=0.27 \pm 0.18 \mathrm{~h}, \mathrm{t} 1 / 2(\beta)=1.95 \pm 0.54 \mathrm{~h}$, $\operatorname{tmax}=0.64 \pm 0.13 \mathrm{~h}$ [44]. Luteolin and its metabolites preferred to distribute in the gastrointestine, liver, kidney and lung. Biliary excretion dominated the elimination pathways of the conjugated luteolin [45].
The four targets with high degree were AKT1(15), IL6(14), VEGFA(14), and EGF(13). IL-6 is a multifunctional cytokine, which is the basis for various immune responses and acute phase reactions, and it is related to depression and autonomic nervous system symptoms. IL- 6 is closely related to hypothalamus pituitary adrenal axis hyperactivity, serotonin metabolism disorder, fatigue, anorexia, depression, and autonomic nervous system symptoms [46]. Akt is a serine/threonine protein kinase, which is an important target of PI3K downstream. Akt has three subtypes (AKT1, AKT2, and Akt3), which play an important role in depression. The activity of Akt protein in the brain tissue of patients with severe depression has been significantly reduced, and Akt can enhance the function of hippocampal stem cells and promote the efficacy of antidepressants [47]. AKT1 has attracted much attention in the study of depression, and it is associated with depression, anxiety symptoms, work, activity, and suicidal tendency of patients with depression [48]. Generally, that AKT1 can regulate the

Table 3 Binding energy ( $\mathrm{kcal} / \mathrm{mol}$ ) and RMSD of three components of Epimedium and target proteins

\begin{tabular}{|c|c|c|c|c|c|c|c|c|}
\hline \multirow{2}{*}{$\begin{array}{l}\text { parameter } \\
\text { receptor }\end{array}$} & \multicolumn{4}{|c|}{ Estimated Free Energy of Binding } & \multicolumn{4}{|c|}{ RMSD } \\
\hline & IL6 & VEGFA & AKT1 & EGF & IL6 & VEGFA & AKT1 & EGF \\
\hline \multicolumn{9}{|l|}{ ligand } \\
\hline luteolin & -5.48 & -6.74 & -6.95 & -7.33 & 0.062 & 0.063 & 0.067 & 0.066 \\
\hline quercetin & -5.2 & -7.31 & -6.95 & -6.3 & 1.165 & 1.166 & 1.17 & 1.17 \\
\hline kaempferol & -5.9 & -6.85 & -6.82 & -6.49 & 0.064 & 0.066 & 0.069 & 0.069 \\
\hline fluoxetine & -4.77 & -5.11 & -5.35 & -5.71 & 0.653 & 0.632 & 0.643 & 0.627 \\
\hline
\end{tabular}


Table 4 Interaction between ligands and target proteins

\begin{tabular}{|c|c|c|c|c|c|c|c|c|}
\hline \multirow{2}{*}{$\frac{\text { Force }}{\text { receptor }}$} & \multicolumn{4}{|l|}{ electrostatic force } & \multicolumn{4}{|c|}{ van der Waals force } \\
\hline & IL6 & VEGFA & AKT1 & EGF & IL6 & VEGFA & AKT1 & EGF \\
\hline \multicolumn{9}{|l|}{ ligand } \\
\hline luteolin & $\begin{array}{l}\text { SER C:227、TRP C: } \\
\text { 225、ASN C:226、ASP } \\
\text { C:221、PRO C: } \\
\text { 222、HIS C:223、SER } \\
\text { E:55、ARG E:57、ALA } \\
\text { E:105、CYS E: } \\
\text { 104、ARG E:103、SER } \\
\text { E:103、ILE E:102、SER } \\
\text { C:228、TYR E:52、TYR } \\
\text { E:59 }\end{array}$ & $\begin{array}{l}\text { CYS A: } 104, \text { HIS } \\
\text { C: } 8, \text { Val C: } 9 \\
\text { TYR A: } 25, \text { CYS } \\
\text { A: } 26, \text { HIS A: } 27, \\
\text { GLN A: } 22, \text { NLE } \\
\text { C: } 10\end{array}$ & $\begin{array}{l}\text { HIS A: 207, TYR } \\
\text { A: } 263, \text { SER A: } \\
\text { 205, LYS A: } 268, \\
\text { ASN A: } 269, \\
\text { ASN A: } 53, \text { TRP } \\
\text { A: } 80, \text { ASP A: } \\
\text { 292, THR A: } 211\end{array}$ & $\begin{array}{l}\text { GLY A: } 12, \text { HIS } \\
\text { A: } 10, \text { ASP A: } \\
11, \text { CYS A: } 14 \\
\text { TYR A: } 13, \text { LEU } \\
\text { A: 8, PRO A: 7, } \\
\text { CYS A: } 6, \text { ASP A: } \\
17\end{array}$ & TYR E: 59 & $\begin{array}{l}\text { GLU A: } 103, \\
\text { CYS A: } 102, \\
\text { PRO A: } 28, \\
\text { ARG A: } 23\end{array}$ & $\begin{array}{l}\text { GLN A: 79, } \\
\text { LEU A: } \\
264, \text { ARG } \\
\text { A: } 206, \\
\text { LEU A: } \\
210, \text { MET } \\
\text { A; } 227, \\
\text { VAL A: } 210\end{array}$ & $\begin{array}{l}\text { GYS A: } \\
\text { 20, VAL } \\
\text { A: } 19 \\
\text { GLY A: } \\
18\end{array}$ \\
\hline quercetin & $\begin{array}{l}\text { CYS V: } 104, \text { ARG V: } 57, \\
\text { SER V: 55, HIS D: 223, } \\
\text { PRO D: 222, ASP D: } \\
\text { 221, TRP D: 225, SER D: } \\
\text { 227, TYR V: 52, PHE D: } \\
\text { 229, SER D: 228, GLN } \\
\text { A: 75, ILE V: } 102, \text { SER V: } \\
101\end{array}$ & $\begin{array}{l}\text { CYS A: } 60, \text { ASN } \\
\text { A: } 62, \text { ASP A: } 63, \\
\text { CYS A: 61, LYS B: } \\
\text { 48, PHE B: } 47 \\
\text { GLU A: } 64, \text { LYS } \\
\text { A: } 107, \text { CYS A: } \\
68, \text { GLY A: } 59\end{array}$ & $\begin{array}{l}\text { GLN A: 59, ILE } \\
\text { A: } 186, \text { LEU A: } \\
\text { 78, ALS A: 58, } \\
\text { CYS A: 60, CYS } \\
\text { A: 77, GLN A: } \\
79, \text { ASN A: } 53 \\
\text { SER A: } 56\end{array}$ & $\begin{array}{l}\text { CYS B: } 14, \text { GLY } \\
\text { B: } 18, \text { ASP B: } 17, \\
\text { GLY B: } 12, \text { ASP } \\
\text { B: } 11, \text { LEU B: } 8 \\
\text { PRO B: } 7, \text { CYS B: } \\
\text { 20, TYR B: } 13\end{array}$ & $\begin{array}{l}\text { ASN D: } \\
\text { 226, Tyr } \\
\text { V: } 59\end{array}$ & $\begin{array}{l}\text { GLU A: } 67, \\
\text { LEU a: } 66, \\
\text { PRO B: } 49, \text { ILE } \\
\text { B: } 46\end{array}$ & / & $\begin{array}{l}\text { SER B: } \\
9, \\
\text { VALB: } \\
\text { 19, HIS } \\
\text { B: } 10\end{array}$ \\
\hline kaempferol & $\begin{array}{l}\text { ARG V: 57, TYR V: } 59 \text {, } \\
\text { ARG V: 103, ALA V: } \\
\text { 105, ILE V: 102, CYS V: } \\
\text { 104, SER V: 101, SER D: } \\
\text { 228, TYR V: 52, SER V: } \\
\text { 55, SER D: 227, ASP D: } \\
\text { 221, PRO D: 222, TRP } \\
\text { D: 225, HIS D: } 223\end{array}$ & $\begin{array}{l}\text { TYR B: } 25, \text { CYS B: } \\
\text { 26, VAL D: } 9 \text {,HIS } \\
\text { D:8,GLN B: } \\
\text { 22,CYS B:104,CYS } \\
\text { B:102 }\end{array}$ & $\begin{array}{l}\text { VAL A: 185, CYS } \\
\text { A: 60, GLN A: } \\
\text { 104, ASD A: } \\
\text { 108, GLN A: 61, } \\
\text { LEU A: 78, ARG } \\
\text { A: 76, ILE A: } 186\end{array}$ & $\begin{array}{l}\text { ASP B: 11, CYS } \\
\text { B: 120, GLY B: } \\
\text { 12, ASP B: } 17, \\
\text { GLY B: } 18, \\
\text { CYSB: } 14, \text { LEU B: } \\
\text { 15, TYR B: } 13 \\
\text { PRO B: } 7, \text { LEU B: } \\
8\end{array}$ & $\begin{array}{l}\text { ASN D: } \\
226\end{array}$ & $\begin{array}{l}\text { NLE D:10, GLU } \\
\text { B :103,HIS B } \\
: 27, \text { LYS B } \\
: 101, \text { PRO B:28 }\end{array}$ & $\begin{array}{l}\text { VAL A: } 83 \\
\text { LYS A: } 111 \\
\text { CYS A: } 77\end{array}$ & $\begin{array}{l}\text { SER B: } \\
9, \text { VAL } \\
\text { B: } 19 \\
\text { HIS B: } \\
10\end{array}$ \\
\hline $\begin{array}{l}\text { Fluoxetine(positive } \\
\text { controls) }\end{array}$ & $\begin{array}{l}\text { ASP B:26, ARG B:30, } \\
\text { LYS C: } 252, \text { ASP C : } 253 \\
\text { GLU C:23, LYS B :27 }\end{array}$ & $\begin{array}{l}\text { CYS B:102, HIS B: } \\
27, \text { GLN B:22, } \\
\text { CYS B:26 }\end{array}$ & $\begin{array}{l}\text { ASN A:31, VAL } \\
\text { A:106, THR A: } \\
\text { 105,ASP A:3 }\end{array}$ & $\begin{array}{l}\text { ASP A:17, CYS } \\
\text { A:20, CYS A: } \\
6,1 Y R \text { A:13, ASP } \\
\text { A :11,CYS A:14, } \\
\text { GLY A:18, }\end{array}$ & $\begin{array}{l}\text { SER E :30, } \\
\text { ARG C } \\
: 231, \text { THR } \\
\text { E:31, ARG } \\
\text { B:182, } \\
\text { LEU C } \\
: 254, \text { TYR } \\
\text { E:27 }\end{array}$ & $\begin{array}{l}\text { HIS D:8, VAL } \\
\text { D:9, PRO B:28, } \\
\text { TYR B :25, LYS } \\
\text { B :101, ARG B } \\
: 23, \text { GLU B } \\
: 103, \text { NLE D: } \\
10, \text { CYS B:104, }\end{array}$ & $\begin{array}{l}\text { ASP A:32, } \\
\text { VAL A;7, } \\
\text { GLY A:109, } \\
\text { VAL A:4, } \\
\text { LEU A :110 }\end{array}$ & $\begin{array}{l}\text { LEU A: } \\
15, \text { LEU } \\
\text { A:8, } \\
\text { VAL A: } \\
19, \text { GLY } \\
\text { A :12, } \\
\text { HIS A } \\
: 16\end{array}$ \\
\hline
\end{tabular}

"/" means no amino acid residues

function of antidepressants and contribute to the formation of synaptic plasticity and nerve transmission. Genebased association identifies loci in VEGFA as potential genetic predictors or SSRI therapeutic response in MDD patients [49]. However, limited studies have been conducted about EGF in depression. The results of the present study show that EGF plays a very important role in the treatment of depression. EGF can be used as another target for the treatment of depression, thus providing a new reference and ideas for the treatment of depression. The possible key components and targets of anti-depression of Epicedium are listed above.

The results of molecular docking showed a good binding activity between the three most important components (luteolin, quercetin, and kaempferol) and the four important target proteins (IL6, VEGFA, AKT1, EGF), and the main forms of interaction between components and targets are electrostatic and van der Waals force. Quercetin, kaempferol, and luteolin possess anti-depression effects, indicating that the research method is reasonable and feasible.
Pathway analysis suggested that Epicedium may play an antidepressant role by regulating TNF signaling pathway, dopaminergic synapse, and prolactin signaling pathway. TNF, dopamine, calcium signaling pathway, prolactin, and I-kappaB kinase/NF-kappaB signaling play important roles in the pathogenesis of depression [5052]. As a messenger, $\mathrm{Ca}^{2+}$ is involved in the regulation of various neural cell functions, such as the release of neurotransmitters, the construction of cells, and the activation of enzyme system. In the central nervous system, slight changes in $\mathrm{Ca}^{2+}$ can lead to significant changes in the function of nerve cells. Therefore, $\mathrm{Ca}^{2+}$ balance is an important factor to maintain the structural integrity and normal function of nerve cells. The increase of $\mathrm{Ca}^{2+}$ in hippocampal neurons can lead to neuronal apoptosis. Calcium imbalance leads to neuronal apoptosis, then changes of hippocampal structure and function, and eventually leads to depression.

Dopamine, as a monoamine transmitter, is a key neurotransmitter in hypothalamus and pituitary gland. 


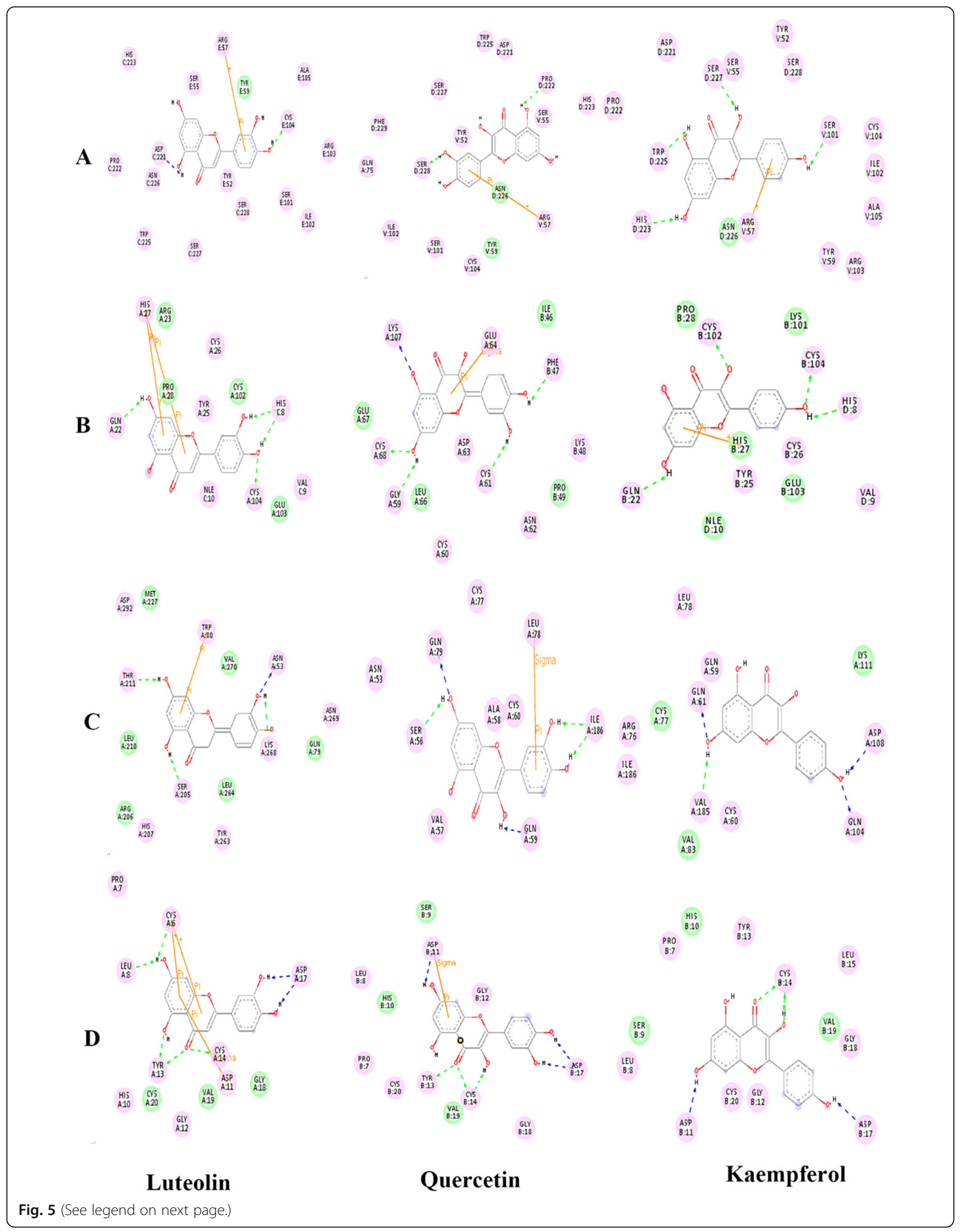


(See figure on previous page.)

Fig. 5 Binding mode of protein and different ligands.(A) Binding mode of IL6 with luteolin, quercetin, and kaempferol.(B) Binding mode of VEGFA with luteolin, quercetin, and kaempferol. (C) Binding mode of AKT1 with luteolin, quercetin, and kaempferol. (D) Binding mode of EGF with luteolin, quercetin, and kaempferol. Pink represents the amino acids that form electrostatic force, and green represents the amino acids that form van der Waals force

The synthesis, release, reuptake, or metabolic disorders of dopamine can lead to depression. Dopamine deficiency can downregulate the dopamine transporter but upregulate the concentration of $\mathrm{D} 2 / 3$ receptor. The decrease of dopamine transporter in amygdala and the increase of dopamine D2/3 receptor in patients with depression indicate that depression may be related to dopamine deficiency in brain. Hence, dopamine plays an important role in the pathophysiological process of depression. The decrease of dopaminergic neurons and the dysfunction of dopaminergic receptors are risk factors for depression. Tumor necrosis factor has two forms, namely, TNF- $\alpha$ and TNF- $\beta$. Its physiological effects are mediated by TNF-R1 and TNF-R2. Serum TNF- $\alpha$ levels in patients with depression significantly increase [53]. In an animal experiment, significant depressive behavior was induced by injecting TNF- $\alpha$ or LPS into the lateral ventricle, Reichenberg [54] found that TNF- $\alpha$ can induce depression and cognitive function changes in humans. In addition, various antidepressants can reduce the level of TNF- $\alpha$ in the peripheral blood of patients with depression. The expression of TNF- $\alpha$ in the dorsolateral prefrontal cortex of patients with severe depression is significantly increased [55]. In addition, Pandey GN found that the expression of TNF- $\alpha$ mRNA and protein in the prefrontal cortex of patients with depression has increased. The serum TNF- $\alpha$ levels can also assess the severity of depression. Therefore, the increase of TNF- $\alpha$ level can lead to the occurrence of depressive symptoms.

Furthermore, more genes were involved in the formation of nerves and synapses. Synapses are the basic structure of information transmission between neurons. They adapt to stimuli by constantly modifying neural connections and circuits. Therefore, synaptic plasticity is the main manifestation of neural plasticity. Depression and other psychological disorders are usually associated with decreased synaptic plasticity in the hippocampus.

Estrogen can affect brain function directly through the estrogen receptor in the brain region, and it plays an important role in depression. It can increase the concentration of 5-hydroxytryptamine, dopamine, norepinephrine, and other neurotransmitters in synapses and affect their release and reuptake. Aged female mice show increased anti-anxiety and -depressant effects after administration of estrogen [56]. In addition, ketamine and its active metabolites have similar affinity to ER $\alpha$ [57]. Ovariectomy can induce depression-like behavior in rats. The implantation of estradiol pellets into both sides of medial amygdala of ovariectomized rats can significantly reduce depressive behavior in rats. This effect may be achieved by activating $\operatorname{Er} \beta$, because $\operatorname{Er} \beta$ agonist can also reverse the depression-like behavior of ovariectomized rats [58]. IL-1 can initiate various immune responses, such as fever, prostaglandin synthesis, neutrophil aggregation and activation, activation of $\mathrm{T}$ cells and $\mathrm{B}$ cells, and production of cytokines [59]. Animal studies on depression have found that the spleen cell of depression rats induced by chronic mild stimulation have increased the production of IL-1. IL includes 11 factors such as IL-1 $\alpha$ and IL-1 $\beta$, among which IL- $1 \alpha$ is widely expressed in many kinds of cells and can be activated without any processing [60]. IL-1 $\beta$ is mainly expressed in bone marrow cells. The activation of pattern recognition receptor may be a biological target for innovative treatment of depression. Dahl [59] found that serum IL-1 $\beta$ in patients with depression is significantly higher than that in healthy control group. Huang [60] found that behavioral depression is induced in rats after the intraventricular injection of IL-1 $\beta$. The possible mechanism is that IL$1 \beta$ activates IL-1 receptor type I in hippocampal neural stem cells, which affect nuclear factor kappaB signal transduction pathway, reduce the proliferation of hippocampal cells, and then lead to depression [61]. The loss and overexpression of IL-1 $\beta$ receptor antagonist in the brain of mice can resist the decrease of hippocampal nerve regeneration related to stress, making it less prone to depression [62].

In this study, we applied the method of network pharmcalogy to to explore the antidepressant mechanism of Epimedium for the first time, and docking verification were added to the network pharmacology as complement to predict the drug targets. Protein-protein interaction and KEGG enrichment analysis results show that prolactin signaling pathway and EGF play an important role in the treatment of depression. There are few studies on this aspect. According to the results of this study, we can carry out research on prolactin signaling pathway and EGF in the future depression research.

\section{Conclusion}

System prediction was used to identify Epicedium with the potential effect on anti-depression. The network pharmacology, a multi-component and multi-targets analysis fit to the TCM treatment, predicted the potential targets and mechanism of formula, and our previous research shows that Epimedium extract has good 
antidepressant effect. This research indicated that prolactin signaling pathway and EGF play an important role in the treatment of depression. The follow-up study could discuss through the regulation of prolactin signaling pathway and EGF in the treatment of depression, which must be thoroughly tested in vivo and in vitro.

\section{Abbreviations}

PPI: protein-protein interaction; GO: Gene Ontology; KEGG: Kyoto Encyclopedia of Genes and Genomes; SPT: Sucrose Preference Test; FST: Forced Swimming Test; TCMSP: Traditional Chinese Medicine Systems Pharmacology; LPS: Lipopolysaccharide; MDD: Major Depressive Disorder; EGF: Epidermal Growth Factor; VEGFA: Vascular endothelial growth factor A IL6: Interleukin-6; AKT1: RAC-alpha serine/threonine-protein kinase

\section{Supplementary Information}

The online version contains supplementary material available at https://doi. org/10.1186/s12906-021-03389-w.

Additional file 1: Supplementary Figure 1. The four targets were IL6 (PDB ID: 5fuc), VEGFA (PDB ID: 6d3o), AKT1(PDB ID: 6s9x), and EGF (PDB ID:1j19). The top three compounds were leteolin, quercetin, and kaempferol. The binding energies of ligand compounds and protein receptors were as follows. The binding energy ranges from $-7.33 \mathrm{kcal} /$ $\mathrm{mol}$ to $-5.20 \mathrm{kcal} / \mathrm{mol}$. Structural model of active ingredients with hub targets. (A) Structural model of IL6 with luteolin, quercetin, and kaempferol. (B) Structural model of VEGFA with luteolin, quercetin, and kaempferol.(C) Structural model of AKT1 with luteolin, quercetin, and kaempferol. (D) Structural model of EGF with luteolin, quercetin, and kaempferol. Supplementary Figure 2. Binding site of active ingredients with hub targets.(A) Binding site of IL6 with luteolin, quercetin, and kaempferol.(B) Binding site of VEGFA with luteolin, quercetin, and kaempferol. (C) Binding site of AKT1 with luteolin, quercetin, and kaempferol. (D) Binding site of EGF with luteolin, quercetin, and kaempferol.

\section{Acknowledgements}

The authors acknowledge gratitude to all the staff who participated in the study.

\section{Authors' contributions}

YKD and BT participated in the design of the study and wrote the manuscript. XX, CXF, YTR and HYM collected and analyzed the data. JLZ, FYS SSZ, SL and JPX did the experiment. HL and ZLZ editedthe manuscript. JHZ, $\mathrm{GL}$ and ZFW consulted the relevant literature.All authors read and approved the final manuscript.

\section{Funding}

This work was supported by the National Natural Science Foundation of China(Grants 81873805.

\section{Availability of data and materials}

The datasets used and/or analysed during the current study are available from the corresponding author on reasonable request.

\section{Declarations}

Ethics approval and consent to participate Not applicable.

\section{Consent for publication}

Not applicable.

\section{Competing interests}

The authors declare that there are no competing of interest.

\section{Author details}

${ }^{1}$ Key Laboratory of Resource Biology and Biotechnology in Western China, Ministry of Education, College of Life Sciences, Institute of Maternal and Infant health, Northwest University, Xi'an 710069, Shanxi Province, China. ${ }^{2}$ Department of Orthopaedic, Tianjin Medical University General Hospital, Anshan Road No.154, Tianjin 300052, Heping District, China. ${ }^{3}$ Department of Neonatology, The First Affiliated Hospital of Medical College,Xi'an Jiaotong University, Xi'an 710069, Shanxi Province, China. ${ }^{4}$ Qinba Chinese Medicine Resources R\&D Center, School of Medicine, Ankang University, Ankang 710069, Shanxi Province, China.

Received: 4 November 2020 Accepted: 10 August 2021

Published online: 03 September 2021

\section{References}

1. Scangos KW, Makhoul GS, Sugrue LP, Chang EF, Krystal AD. State-dependent responses to intracranial brain stimulation in a patient with depression. Nature Medicine. Feb 2021;27(2):229-+.

2. Wigner P, Czarny P, Galecki P, Su K-P, Sliwinski T. The molecular aspects of oxidative \& nitrosative stress and the tryptophan catabolites pathway (TRYC ATs) as potential causes of depression. Psychiatry Research. 2018;262:56674.

3. Cui Y, Cao K, Lin H, et al. Early-Life Stress Induces Depression-Like Behavior and Synaptic-Plasticity Changes in a Maternal Separation Rat Model: Gender Difference and Metabolomics Study. Frontiers in Pharmacology. Feb 26 2020;11.

4. Qu S-Y, Li X-Y, Heng X, et al. Analysis of Antidepressant Activity of HuangLian Jie-Du Decoction Through Network Pharmacology and Metabolomics. Frontiers in Pharmacology. Mar 4 2021;12.

5. Wang $\mathrm{C}$, Lin $\mathrm{H}$, Yang $\mathrm{N}$, et al. Effects of Platycodins Folium on Depression in Mice Based on a UPLC-Q/TOF-MS Serum Assay and Hippocampus Metabolomics. Molecules. May 1 2019;24(9).

6. Du H, Zhao H, Lai X, et al. Metabolic profiles revealed synergistically antidepressant effects of lilies and Rhizoma Anemarrhenae in a rat model of depression. Biomedical Chromatography. Jul 2017;31(7).

7. Short B, Fong J, Galvez V, Shelker W, Loo CK. Side-effects associated with ketamine use in depression: a systematic review. Lancet Psychiatry. Jan 2018;5(1):65-78.

8. Andrade C. Ketamine for Depression, 1: Clinical Summary of Issues Related to Efficacy, Adverse Effects, and Mechanism of Action. Journal of Clinical Psychiatry. Apr 2017;78(4):E415-9.

9. Cao H, Zuo C, Huang Y, et al. Hippocampal proteomic analysis reveals activation of necroptosis and ferroptosis in a mouse model of chronic unpredictable mild stress-induced depression. Behavioural Brain Research. Jun 11 2021;407.

10. Xiong Z, Yang J, Huang Y, et al. Serum metabonomics study of antidepressive effect of Xiao-Chai-Hu-Tang on rat model of chronic unpredictable mild stress. Journal of Chromatography B-Analytical Technologies in the Biomedical and Life Sciences. Sep 1 2016;1029:28-35.

11. Huang S, Meng N, Chang B, Quan X, Yuan R, Li B. Anti-inflammatory Activity of Epimedium brevicornu Maxim Ethanol Extract. Journal of Medicinal Food. Jul 2018;21(7):726-33.

12. Zhao $Y$, Chen S, Wang Y, LV C, Wang J, Lu J. Effect of drying processes on prenylflavonoid content and antioxidant activity of Epimedium koreanum Nakai. Journal of Food and Drug Analysis. Apr 2018;26(2):796-806.

13. Kou Y, Wang Z, Wu Z, et al. Epimedium Extract Promotes Peripheral Nerve Regeneration in Rats. Evidence-Based Complementary and Alternative Medicine. 2013;2013:2013.

14. Zhang X, Sun H, Su Q, et al. Antidepressant-like activity of icariin mediated by group I mGluRs in prenatally stressed offspring. Brain \& Development. Aug 2017;39(7):593-600.

15. Song X, Zhang Y, Yang N, Dai E, Wang L, Du H. Molecular mechanism of celastrol in the treatment of systemic lupus erythematosus based on network pharmacology and molecular docking technology. Life Sciences. Jan 1 2020;240.

16. Li T, Zhang W, Hu E, et al. Integrated metabolomics and network pharmacology to reveal the mechanisms of hydroxysafflor yellow A against acute traumatic brain injury. Computational and structural biotechnology journal. 2021 2021;19:1002-1013.

17. Zhong Y, Luo J, Tang T, et al. Exploring Pharmacological Mechanisms of Xuefu Zhuyu Decoction in the Treatment of Traumatic Brain Injury via a Network Pharmacology Approach. Evidence-Based Complementary and Alternative Medicine. 2018;2018:2018. 
18. Ma C, Xu T, Sun X, et al. Network Pharmacology and Bioinformatics Approach Reveals the Therapeutic Mechanism of Action of Baicalein in Hepatocellular Carcinoma. Evidence-Based Complementary and Alternative Medicine. 2019;2019.

19. Zhu W, Fan X, Wei H, et al. Mechanism Research of Apatinib-Treated Breast Cancer Based on Network Pharmacology. Chinese Pharmaceutical Journal. 2016 2016;51(18):1569-1573.

20. Liu Y, Ju Y, Qin X. Studies on the compatibility mechanism and material basis of Danggui Buxue Decoction against anemia mice using metabonomics and network pharmacology. The Journal of pharmacy and pharmacology. 2021-Apr-27 2021;73(6):767-777.

21. Gong P, Guo Y, Li X, Wang N, Gu J. Exploring active compounds of Jinhua Qinggan Granules for prevention of COVID-19 based on network pharmacology and molecular docking. Chinese Traditional and Herbal Drugs. 2020 2020;51(7):1685-1693.

22. Ru J, Li P, Wang J, et al. TCMSP: a database of systems pharmacology for drug discovery from herbal medicines. Journal of Cheminformatics. Apr 16 2014;6.

23. Xu X, Zhang W, Huang C, et al. International Journal of Molecular Sciences. Jun 2012;13(6):6964-82.

24. Walters WP, Murcko MA. Prediction of 'drug-likeness'. Advanced Drug Delivery Reviews. Mar 31 2002:54(3):255-271.

25. Feng $W$, Ao $H$, Yue $S$, Peng $C$. Systems pharmacology reveals the unique mechanism features of Shenzhu Capsule for treatment of ulcerative colitis in comparison with synthetic drugs. Scientific Reports. Nov 1 2018;8.

26. von Mering $C$, Jensen $L$, Snel B, et al. STRING: known and predicted protein-protein associations, integrated and transferred across organisms. Nucleic Acids Research. Jan 1 2005;33:D433-7.

27. Huang DW, Sherman BT, Lempicki RA. Systematic and integrative analysis of large gene lists using DAVID bioinformatics resources. Nature Protocols. 2009 2009:4(1):44-57.

28. Chen L, Zhang Y-H, Wang S, Zhang Y, Huang T, Cai Y-D. Prediction and analysis of essential genes using the enrichments of gene ontology and KEGG pathways. Plos One. Sep 5 2017;12(9).

29. Adams R, Burnley RJ, Valenzano CR, et al. Discovery of a junctional epitope antibody that stabilizes IL-6 and gp80 protein: protein interaction and modulates its downstream signaling. Scientific Reports. Jan 30 2017:7.

30. Quambusch L, Landel I, Depta L, et al. Covalent-Allosteric Inhibitors to Achieve Akt Isoform-Selectivity. Angewandte Chemie-International Edition. Dec 19 2019;58(52):18823-18829.

31. Lu HS, Chai JJ, Li M, Huang BR, He CH, Bi RC. Crystal structure of human epidermal growth factor and its dimerization. Journal of Biological Chemistry. Sep 14 2001;276(37):34913-34917.

32. Hopkins AL. Network pharmacology: the next paradigm in drug discovery. Nature Chemical Biology. Nov 2008;4(11):682-90.

33. Heinrich M, Appendino $G$, Efferth $T$, et al. Best practice in research Overcoming common challenges in phytopharmacological research. Journal of Ethnopharmacology. Jan 10 2020;246.

34. Sasaki K, El Omri A, Kondo S, Han J, Isoda H. Rosmarinus officinalis polyphenols produce anti-depressant like effect through monoaminergic and cholinergic functions modulation. Behavioural Brain Research. Feb 1 2013;238:86-94.

35. Akinrinde AS, Adebiyi OE. Neuroprotection by luteolin and gallic acid against cobalt chloride-induced behavioural, morphological and neurochemical alterations in Wistar rats. Neurotoxicology. Sep 2019;74:252-63.

36. Mehta V, Parashar A, Udayabanu M. Quercetin prevents chronic unpredictable stress induced behavioral dysfunction in mice by alleviating hippocampal oxidative and inflammatory stress. Physiology \& Behavior. Mar 15 2017;171:69-78

37. Khan A, Ali T, Rehman SU, et al. Neuroprotective Effect of Quercetin Against the Detrimental Effects of LPS in the Adult Mouse Brain. Frontiers in Pharmacology. Dec 11 2018;9.

38. Liang Y, Tan Y, Zhang S, Wang S, Wang L, Yang Y. Effect and mechanism of kaempferol on depression-like behavior in elderly rats with chronic stress depression. The Chinese Journal of Clinical Pharmacology. 2020 2020;36(24):40284030.

39. Zhu S, Lei S, Zhou S, et al. Luteolin shows antidepressant-like effect by inhibiting and downregulating plasma membrane monoamine transporter (PMAT, Slc29a4). Journal of Functional Foods. Mar 2019;54:440-8.

40. Samad N, Saleem A, Yasmin F, Shehzad MA. Quercetin Protects Against Stress-Induced Anxiety- and Depression-Like Behavior and Improves Memory in Male Mice. Physiological Research. 2018 2018;67(5):795-808.
41. Zeng C, Mi S, Luo S, Huang T, Hong X, Luo Y. Pharmacokinetic Studies of Quercetin in Semen Cuscutae. Traditional Chinese Drug Research and Clinical Plarmacology. 2004 2004;15(1):37-40.

42. Dongfang WU, Lei Z, Siping Z. Distribution of quercetin in plasma and tissues in rats. Chinese Journal of Hospital Pharmacy. 2008 2008;28(21):1822-1824.

43. Zhang M, Kong L, Luo C, Li X, Zhou Y. Pharmacokinetic Study of Keampferol in Rabbits. China Pharmacy. 2014 2014;25(43):4040-4042.

44. Ying J-Y, Ma J-L, Xia Z-L, Yao T-W. Pharmacokinetics of luteolin from Elsholtzia blanda extracts in rats. Yao xue xue bao $=$ Acta pharmaceutica Sinica. 2008-May 2008;43(5):523-527.

45. Deng C, Gao C, Tian X, et al. Pharmacokinetics, tissue distribution and excretion of luteolin and its major metabolites in rats: Metabolites predominate in blood, tissues and are mainly excreted via bile. Journal of Functional Foods. Aug 2017;35:332-40.

46. Saeidienik F, Shahraki MR, Fanaei H, Badini F. Research Paper: The Effects of Iron Oxide Nanoparticles Administration on Depression Symptoms Induced by LPS in Male Wistar Rats. Basic and Clinical Neuroscience. May-Jun 2018;9(3):209-15.

47. Meyer KD, Morris JA. Immunohistochemical analysis of Disc1 expression in the developing and adult hippocampus. Gene Expression Patterns. Sep 2008;8(7-8):494-501.

48. Yang C, Sun N, Ren Y, et al. Association between AKT1 gene polymorphisms and depressive symptoms in the Chinese Han population with major depressive disorder. Neural Regeneration Research. Jan 2012;7(3):235-9.

49. Kao C-F, Liu Y-L, Yu YWY, et al. Gene-based analysis of genes related to neurotrophic pathway suggests association of BDNF and VEGFA with antidepressant treatment-response in depressed patients. Scientific Reports. May 3 2018;8.

50. Cao X, Li L-P, Wang Q, et al. Astrocyte-derived ATP modulates depressivelike behaviors. Nature Medicine. Jun 2013;19(6):773-+

51. Szpunar MJ, Parry BL. A systematic review of cortisol, thyroid-stimulating hormone, and prolactin in peripartum women with major depression. Archives of Womens Mental Health. Apr 2018;21(2):149-61.

52. Li C, Xu X, Wang Z, et al. Exercise ameliorates post-stroke depression by inhibiting PTEN elevation-mediated upregulation of TLR4/NF-kappa B/ NLRP3 signaling in mice. Brain Research. Jun. 1736;1:2020.

53. Trivedi MH, Rush AJ, Wisniewski SR, et al. Evaluation of outcomes with citalopram for depression using measurement-based care in STAR*D: Implications for clinical practice. American Journal of Psychiatry. Jan 2006;163(1):28-40.

54. Reichenberg A, Yirmiya R, Schuld A, et al. Cytokine-associated emotional and cognitive disturbances in humans. Archives of General Psychiatry. May 2001;58(5):445-52

55. Nickola TJ, Ignatowski TA, Reynolds JL, Spengler RN. Antidepressant druginduced alterations in neuron-localized tumor necrosis factor-alpha mRNA and alpha(2)-adrenergic receptor sensitivity. Journal of Pharmacology and Experimental Therapeutics. May 2001;297(2):680-7.

56. Farzaneh S, Zarghi A. Estrogen Receptor Ligands: A Review (2013-2015). Scientia Pharmaceutica. Sep 2016;84(3):409-27.

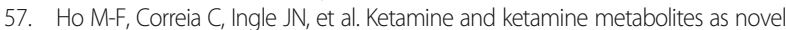
estrogen receptor ligands: Induction of cytochrome P450 and AMPA glutamate receptor gene expression. Biochemical Pharmacology. Jun 2018;152:279-92.

58. Morales T, Lorenson M, Walker AM, Ramos E. BOTH PROLACTIN (PRL) AND A MOLECULAR MIMIC OF PHOSPHORYLATED PRL, S179D-PRL, PROTECT THE HIPPOCAMPUS OF FEMALE RATS AGAINST EXCITOTOXICITY. Neuroscience. Jan 31 2014:258:211-7.

59. Dinarello CA. A clinical perspective of IL-1 beta as the gatekeeper of inflammation. European Journal of Immunology. May 2011;41(5):1203-17.

60. van de Veerdonk FL, Netea MG. New insights in the immunobiology of IL-1 family members. Frontiers in Immunology. 2013;2013:4.

61. Koo JW, Duman RS. IL-1 beta is an essential mediator of the antineurogenic and anhedonic effects of stress. Proceedings of the National Academy of Sciences of the United States of America. Jan 15 2008;105(2):751-756.

62. Goshen I, Kreisel T, Ben-Menachem-Zidon O, et al. Brain interleukin-1 mediates chronic stress-induced depression in mice via adrenocortical activation and hippocampal neurogenesis suppression. Molecular Psychiatry. Jul 2008;13(7):717-28.

\section{Publisher's Note}

Springer Nature remains neutral with regard to jurisdictional claims in published maps and institutional affiliations. 